\title{
An on-line sample pretreatment technique for the HPLC analysis of plant samples.
}

\begin{abstract}
A continuous-flow, on-line sample pretreatment technique using a silica gel microsyringe extractor has been developed. All steps including extraction, separation, clean-up, and concentration occur in the microsyringe. The overall sample pretreatment process takes $<10$ min per sample. Different polarity chemicals in the plant sample are successively extracted and separated, and analyzed in parallel using HPLC-UV and HPLC-UV-MS/MS. Polycyclic aromatic hydrocarbons, alkylphenols, and plant hormones were determined as model compounds for nonpolar, intermediate polarity, and polar fractions, respectively. All the parameters that influence the extraction and separation efficiency of the microsyringe extractor have been optimized and evaluated. Under the optimized conditions, recoveries of target compounds ranged from 78.4 to $101.9 \%$, the RSD was $<12.8 \%$ and the square of the correlation coefficient was $>0.99$. Complex plant samples of Sambucus Mandshurica Kitag have been tested using this method. Fluorene, phenanthrene, pyrene, and plant hormones were detected in all the samples, and concentrations ranged from 24.2-34.9, 43.8-67.1, 25.929.2 , and $14.5 \sim 110.8 \mathrm{ng} / \mathrm{g}$, respectively.
\end{abstract}

Keyword: HPLC-UV-MS; Microsyringe extraction; On-line analysis; Sample preparation; Sample pretreatment. 